\title{
Defect Tolerant Perovskite Solar Cells from Blade coated Non-Toxic Solvents
}

\author{
Zhuoneng Bi, ${ }^{1,2}$ Xabier Rodríguez-Martínez, ${ }^{3}$ Clara Aranda, ${ }^{1}$ Enrique Pascual San \\ José, ${ }^{3}$ Alejandro R. Goñi, ${ }^{3,4}$ Mariano Campoy-Quiles, ${ }^{3 *}$ Xueqing $\mathrm{Xu},{ }^{2 *}$ Antonio \\ Guerrero $^{1 *}$
}

${ }^{1}$ Institute of Advanced Materials (INAM), Universitat Jaume I, 12006 Castelló, Spain

${ }^{2}$ Key Laboratory of Renewable Energy, Guangdong Key Laboratory of New and Renewable Energy Research and Development, Guangzhou Institute of Energy Conversion, Chinese Academy of Sciences, Guangzhou 510640, China

${ }^{3}$ Institut de Ciència de Materials de Barcelona (ICMAB-CSIC), Campus de la UAB, 08193 Bellaterra, Spain

${ }^{4}$ ICREA, Passeig Lluís Companys 23, 08010 Barcelona, Spain

Email: xuxq@ms.giec.ac.cn, mcampoy@icmab.es; $\underline{\text { aguerrer@uji.es }}$

7 February 2019

\begin{abstract}
Understanding crystallization of lead halide perovskites by industrially relevant techniques using non-toxic solvents is a topic that needs development. To this date, highest efficiency devices are prepared by deposition of the perovskite layer using nonscalable techniques, toxic solvents and/or require additional processing steps. In this work, we show that efficient one-step perovskite solar cells can be obtained by doctor blade. The perovskite film is formed under supersaturation regime from non-toxic solvents following spherulitic growth. This method results in highly crystalline perovskite films with preferential crystal orientation. Co-local photoluminescence and light-beam induced current experiments show that generated chemical defects are confined at the boundary of spherulites and these do not have a negative effect on the extracted photocurrent. Strikingly, spherulitic formation, rather than being detrimental, can lead to better photovoltaic performance in hybrid perovskite films. This is further confirmed in photovoltaic devices with record efficiencies of $18.0 \%$ for $\mathrm{MAPbI}_{3}(\mathrm{MA}=$ Methyl ammonium) for doctor bladed processing using non-toxic solvents. Moreover, large area devices $\left(1.53 \mathrm{~cm}^{2}\right)$ fabricated using doctor blade show remarkable efficiencies $(14.2 \%)$ reinforcing the viability of this solar technology towards industrialization.
\end{abstract}

Keywords: perovskite, spherulite, defect tolerance, doctor blade, non-toxic solvent. 


\section{Introduction}

Hybrid organic-inorganic perovskite materials have recently emerged as a serious alternative for high-power, conversion-efficient (PCE) photovoltaic devices at low costs. ${ }^{1,2}$ Photoactive perovskite films can be produced by depositing precursor inks from solution, which represents a real economic advantage over commercially available photovoltaic technologies relying on high vacuum processes. Recently, impressive record efficiencies of $\sim 22 \%$ have been reported at lab scale for small area (i.e. $0.16 \mathrm{~cm}^{2}$ ) using an industrially non-scalable technique, namely spin coating. ${ }^{1,3}$ Unfortunately, highest efficiency devices are typically produced with toxic solvents such as N,Ndimethylformamide (DMF) and require further steps to improve the uniformity of the films and reduce chemical defects. ${ }^{4-6}$ Alternatives to spin coating and to DMF are being searched that meet the stringent requirements demanded by large scale production.

Different industrially compatible deposition techniques like doctor blade, ${ }^{7-11}$ inkjet printing ${ }^{12}$, slot die $^{13}$ or spray coating ${ }^{14,15}$ have been employed with relative success, achieving efficiencies ranging from $10 \%$ to $18 \%$. From these techniques, doctor blade is a promising lab-scale deposition method since the rheological properties of the precursor solution, the drying dynamics as well as the mechanical shearing can be partially transferred to other continuous-like deposition techniques such as slot die coating. ${ }^{8-11}$ In doctor blade, the precursor solution is placed between the edge of the blade and the substrate. Then, the substrate (or the blade) is unidirectionally moved spreading homogeneously the solution (Figure 1a), leaving a wet thin film on the substrate which dries upon solvent evaporation. Until now, crystallization by doctor blade has been problematic since the kinetics of the growth have not been fully understood, leading to irregular film thickness and/or pinholes. ${ }^{8}, 10$ One strategy to reduce the presence of pinholes has been to increase the thickness of the perovskite layer to values beyond $1 \mu \mathrm{m}$ achieving high efficiencies $(15 \%) .{ }^{9}$ Recently, the problem with the crystallization kinetics has been overcome for doctor bladed devices by means of using an additional step like the anti-solvent extraction. ${ }^{7}$ All these results highlight the fact that crystallization by doctor blade using one-step methods is yet to be fully mastered.

On the other hand, the precursor inks employed in the preparation of lead halide perovskites by doctor blade have been formulated containing the toxic solvent DMF. ${ }^{8,9}$ The role of the DMF in the ink formulation consists in dissolving the lead halide precursor. However, difficulties removing DMF from the films yields non-uniform perovskite films. For this reason, the use of additional steps such as anti-solvent treatment $^{7}$ that washes out the excess solvent is required. A suitable non-toxic alternative to DMF is dimethyl sulfoxide (DMSO). ${ }^{16}$ Removing DMSO from the film is, however, still less favored than removal of DMF due to the higher boiling point of DMSO and its stronger coordination ability towards $\mathrm{Pb}^{17}$ Therefore, further ink formulation development is required in order to meet the industrial standards of safety and environmental protection. 
In this work, we present a method to obtain high quality $\mathrm{MAPbI}_{3}$ under saturation regime by one-step doctor blade in combination with non-toxic solvents. This method produces highly crystalline perovskite films in one step by using inks at the solubility limit of the precursors and relatively high deposition temperatures of $150{ }^{\circ} \mathrm{C}$. The process readily forms highly oriented perovskite layers that crystallize via spherulite growth. The space filling nature of this type of growth means that the gaps between the primary branches of spherulites are filled with smaller branches, thus minimizing the presence of pinholes. Interestingly, we show that chemical defects are mainly confined at the spherulite grain boundaries and these do not have a negative impact on device performance. Device efficiency can be further improved by deposition of the film under dry conditions leading record efficiency of $18.0 \%$ for devices fabricated by doctor blade using non-toxic solvents.

\section{Results and discussion}

\subsection{Ink and Deposition Optimization}

In order to show the potential of the blade coating deposition process, we have chosen to work with the most simple and studied perovskite formulation containing $\mathrm{MAPbI}_{3}$ (MA=Methyl ammonium). The optimization of the film quality involves the precise control of some key parameters, namely the concentration and the deposited volume of the precursor solution, the speed of the moving blade, the height of the gap and the temperature of the substrate. Moreover, the solvent formulation is important since it must contain a highly polar solvent to fully dissolve the lead halide $\left(\mathrm{PbI}_{2}\right)$. DMF, DMSO and their mixtures are the main choice published in literature. As crystallization of the perovskite takes place via intermediate coordination complexes ( $\left.\mathrm{MAPbI}_{3} \cdot \mathrm{DMSO}\right)$
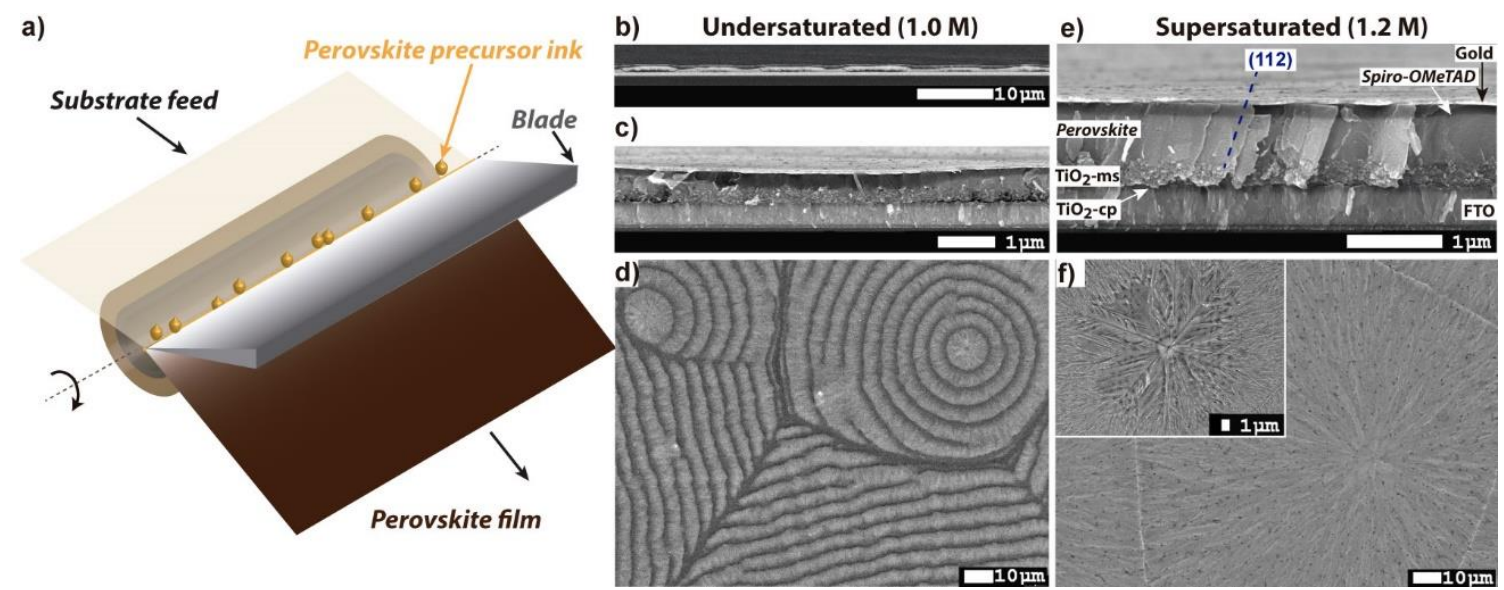

Figure 1: a) Schematic diagram of the doctor blade set up under operation conditions to produce $\mathrm{MAPbI}_{3}$ films. SEM images of films and complete devices fabricated using undersaturated conditions b-d) and saturated conditions e-f). The inset in f) shows a representative spherulite-type domain. b,c and e) are cross-sectional view images at different magnifications. d) and f) are top view images. 
it is clear that the coordinating chemistry nature of the solvents will determine the crystallization kinetics. ${ }^{4,} 18,19$ In our work, DMSO (b.p. $190{ }^{\circ} \mathrm{C}$ ) was selected as solubilizing solvent for $\mathrm{PbI}_{2}$ as it adequately assists the crystallization of the perovskite. ${ }^{20}$ One negative effect of using the required highly coordinating solvents is its trapping within the perovskite film, which makes difficult its removal. ${ }^{18}$ In addition, a secondary solvent like $\gamma$-butyrolactone (GBL) is introduced to control the solvent evaporation rate and crystallization of the perovskite due to its lower coordination ability in comparison to DMSO, allowing the modification of different ink properties simultaneously.

Complete optimization using GBL:DMSO was carried out as described in the supporting information and methods. The best conditions were obtained with a volume ratio of 3:2 (GBL:DMSO), as it provides a good combination between the ability of DMSO to dissolve the $\mathrm{PbI}_{2}$ and the GBL to control the evaporation kinetics. By using a $1 \mathrm{M}$ solution of MAI:PbI 2 in the GBL:DMSO mixture we were able to reproduce the concentric ring morphology (Figure 1d) reported by Huang for a totally different ink formulation containing DMF. ${ }^{10}$ It is not surprising that this structure was reported to produce relatively low PCE $(\sim 12 \%)$ as the dark rings represent areas where the thickness of the perovskite is too thin (Fig. 1b and 1c). Indeed, in photovoltaic devices the Electron Transport Layer $\left(\mathrm{ETL}, \mathrm{TiO}_{2}\right)$ is exposed to the Hole Transport Layer (ETL) in those dark areas leading to charge shunting pathways, as confirmed in the cross section images. Here we note that this type of morphology has been previously observed for different polymers known to follow spherulitic growth like poly(3-hydroxybutyrate) or poly(vinylidene fluoride). ${ }^{21,22}$

Importantly, when the concentration of the perovskite precursors is increased to 1.2 $M$ the solubility limit in this solvent mixture is reached, as observed in this work, and saturation of the ink during crystallization provides high quality perovskite layers in one step. Cross-section SEM image (Fig. 1e) shows highly ordered morphology with regular thickness of $\mathrm{MAPbI}_{3}$ of about $550 \mathrm{~nm}$ on top of the mesoporous $\mathrm{TiO}_{2}$ layer. Importantly, the perovskite layer does not show exposed areas of $\mathrm{TiO}_{2}$, as can be seen in the analysis of large area image scans in cross section (Supporting Information). The top view (Fig. 1f) shows radial fibrous units that arrange around a central crystallization seed with domains of over hundreds of microns. This type of crystal growth is often referred to as spherulitic, and is space filling in nature, only stopping when two spherulites meet each other. During spherulitic growth, impurities are characteristically pushed towards the grain boundaries. Indeed, the spherulites are delimited by grain boundaries observed in the SEM as white lines. These bright edges are likely due to boundaries enriched with heavy atoms such as $\mathrm{Pb}$ or I as confirmed by EDX. In this respect, self-passivation by generation of $\mathrm{PbI}_{2}$ has been reported previously to have a beneficial effect in photovoltaic devices. ${ }^{23}$ The presence of perfectly formed spherulites can be detected in several areas of the film, see inset in Figure 1f. 


\subsection{Structural and Optical properties of the film}

The $\mathrm{MAPbI}_{3}$ crystalline properties of the films fabricated under saturation conditions $(1.2 \mathrm{M})$ were further evaluated by measuring XRD diffraction patterns and were compared with films fabricated by spin coating with the standard antisolvent washing and DMF:DMSO mixtures (Figure 2a). These measurements were performed over the same substrates that were in photovoltaic devices (Glass/FTO/TiO ${ }_{2}-\mathrm{cp} / \mathrm{TiO}_{2}-$ ms/MAPbI ${ }_{3}$ ) in the final section. Both deposition methods exhibit the same crystalline structure for the perovskite. There are, however, differences in crystallite orientation with respect to the substrate. Whilst the perovskite films fabricated by spin coating are highly oriented preferentially over the 110 plane on the substrate, those fabricated by doctor blade are mostly oriented over the 112 plane. Both crystallographic planes are schematically shown in Fig. 2b. The intensity of the peaks related to the 112 plane is much higher than that of the 110 plane. Moreover, these peaks are strongly enhanced in our films compared to other reported doctor bladed devices from DMF solution, which highlights the highly crystalline nature of the prepared samples. ${ }^{9}$ The 112 plane is also shown in the cross-section SEM image (Figure 1e) denoting the preferential cleave of the film on this plane for cross-section sample preparation.
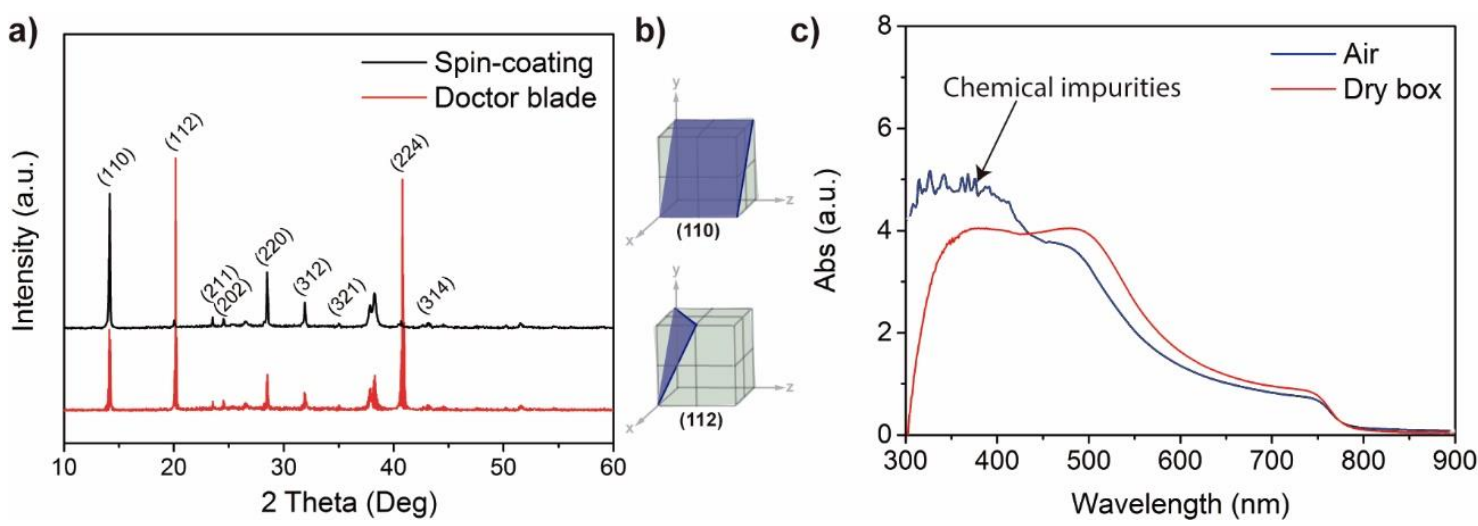

Figure 2: a) XRD diffraction patterns of perovskite films deposited by either spin coating or doctor blade on Glass/FTO/TiO $2-\mathrm{cp} / \mathrm{TiO}_{2}-\mathrm{ms}$. b) Main crystallographic planes observed in a). c) Absorption spectra of $\mathrm{MAPbI}_{3}$ prepared by doctor blade either in air conditions (R.H. $45 \%$ ) or inside a dry box $\left(<0.01\right.$ vol\% $\left.\mathrm{H}_{2} \mathrm{O}\right)$.

We have previously reported that different polyplumbate ions $\left(\mathrm{PbIS}_{5}^{+}, \mathrm{PbI}_{2} \mathrm{~S}_{4}, \mathrm{PbI}_{3} \mathrm{~S}_{3}{ }^{-}\right.$ , $\mathrm{PbI}_{4} \mathrm{~S}_{2}{ }^{2-}, \ldots$ ) are formed during $\mathrm{MAPbI}_{3}$ crystallization if the solvent and ambient conditions are not properly controlled. ${ }^{17}$ These species are detected by absorption measurements in the region of 300-450 $\mathrm{nm}$ and can act as chemical defects having an impact on solar cell performance. ${ }^{17,18}$ Here, we performed absorption measurements to discern whether this type of chemical impurities is present in films prepared by doctor blade. Undercoordinated polyplumbate ions are present in the films prepared under different solvent formulations under ambient conditions, see supporting information. Figure $2 \mathrm{c}$ shows the effect of processing in ambient conditions (under a relative 
humidity of ca. $45 \%$ ), in which case, coordinated plumbate ions are inevitably present. Moreover, we note that the detector is nearly saturated due to the high intensity of the signal and, hence, the signal is noisy in this region. These impurities can be attributed to coordinated complexes with water molecules as they were not detectable when the blade coating was performed inside a glove box (containing less than $0.01 \mathrm{vol} \% \mathrm{H}_{2} \mathrm{O}$ ). In this sense we have recently reported that crystallized water is usually present when devices are processed in R.H. $45 \%$ and are related to the bulk properties of the films. ${ }^{24}$ As discussed below, devices prepared under dry conditions perform better than those prepared under ambient conditions. Therefore, we highlight that not only the morphology of the perovskite layer is important but also the actual chemical impurities present in the film.

\subsection{Electrical properties of Spherulites and chemical defects}

In order to unravel the morphology-performance dependence in the blade coated samples, co-local photoluminescence (PL) and light-beam induced current (LBIC) experiments were performed in fully functional photovoltaic devices. The PL serves to identify the different microstructures and morphologies that coexist in the perovskite film, as well as detecting the presence of defects, typically acting as non-radiative recombination centers for photogenerated carriers. On the other hand, the LBIC signal provides the corresponding local device photocurrent. To do so, we use a $633 \mathrm{~nm} \mathrm{He}-\mathrm{Ne}$ laser to probe the different perovskite morphologies according to the shift and intensity of their main PL peak, while we collect simultaneously the output photocurrent due to the exposure of the device to the focused laser beam. In Figure 3 we compare the data for representative spin coated and blade coated devices, the latter containing spherulites.

The PL intensity maps of spin-coated complete devices (left top image in Figure 3a) exhibit stripes of alternating intensity distributed all over the background of the image. These correspond to thickness oscillations throughout the solution processed perovskite films. While they are not so pronounced, we have also observed small thickness oscillations in the blade coated samples. Spin coated films also exhibit small black spots, which have been previously associated to the Spiro-OMeTAD layer. On the other hand, the spherulites obtained during blade coating are clearly seen in the PL images. Generally speaking, the PL intensity is strongly quenched at defects and particularly at the spherulite borders, where impurities are concentrated. In addition, the shift of the PL peak energy may reflect a slightly different local morphology or stoichiometry. The shifts observed in both types of samples are, however smaller than those observed when changing the phase of the perovskite from tetragonal to cubic. 

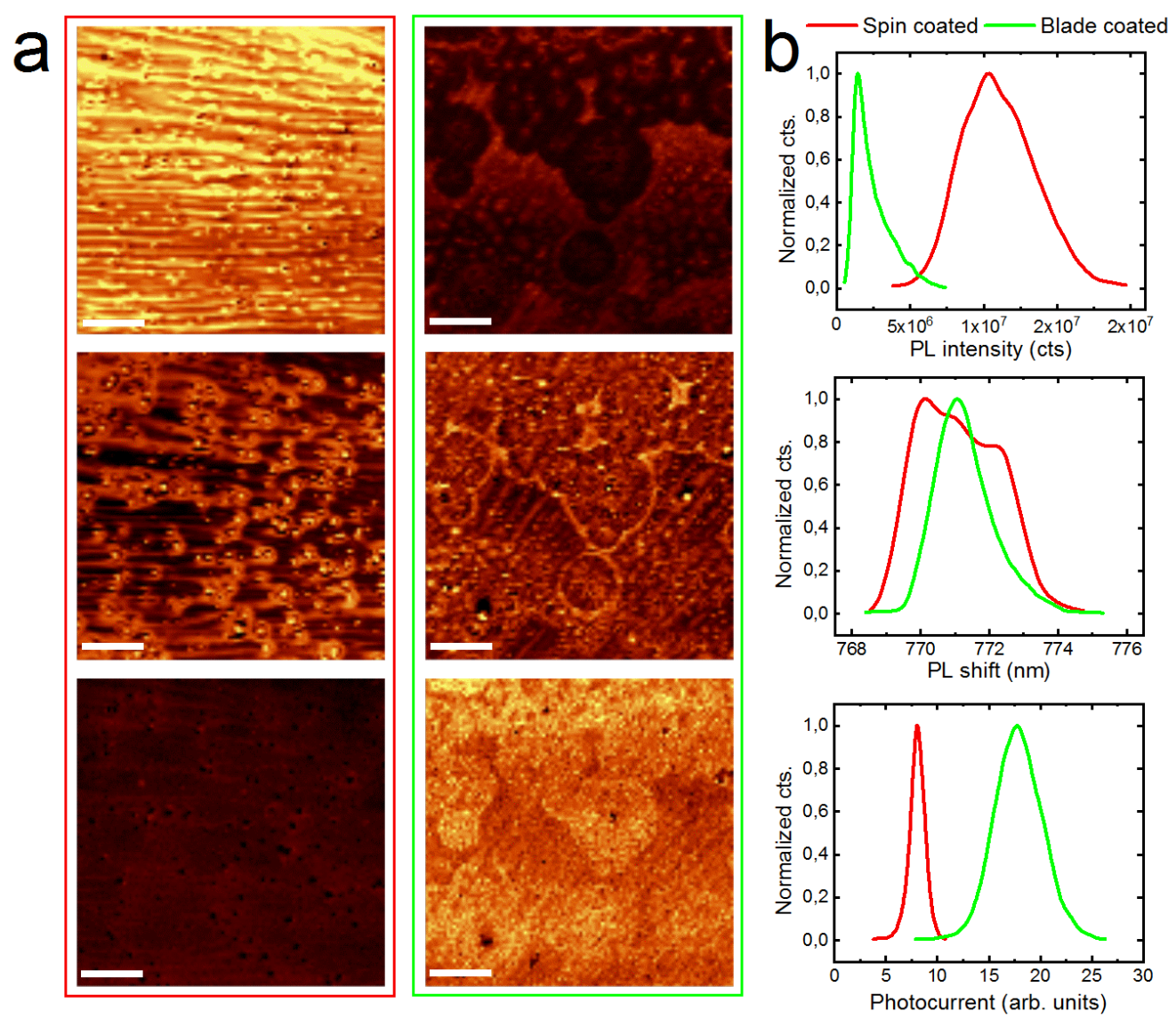

Figure 3. (a) PL and LBIC maps taken in spin coated (red rectangle) and blade coated (green rectangle) perovskite devices. The top images correspond to the integrated PL intensity. The middle images correspond to the location of PL maximum. The bottom images represent the electrical response of the device (photocurrent) upon the exposure to the $633 \mathrm{~nm}$ laser used to measure the PL. The XY scale bar corresponds to 400 microns. The intensity scale was kept constant in each pair of images (integrated PL, PL maximum and photocurrent) to easy the comparison between deposition methods. (b) Statistical distribution of the data presented in the images for the same spin coated (red line) and blade coated (green line) devices. PL intensities as well as photocurrent values were normalized taking into account the incident laser power

The combination of the PL maps with the electrical response of the devices (Figure 3a) clearly shows the location of the defects, which correlate perfectly with the large variations in the PL shift and the spikes (drops) in photocurrent. For the blade coated samples, the spherulites are clearly apparent in the maps of the three monitored magnitudes: PL intensity and maximum position as well as photocurrent. Figure 3b shows the histograms for these three quantities, as obtained from the statistical analysis of the corresponding images, normalized by the incident laser power. In particular, the variations in position of the PL maximum (PL shift) which have several maxima for the spin coated sample, suggest that the microstructure of the perovskite film varies 
significantly. The photocurrent, on the other hand, exhibits a narrower modal distribution as compared to the PL properties. This fact indicates that the perovskite films are rather tolerant to the presence of defects and small morphological variations, which is a key feature towards the industrialization of this technology.

One very interesting observation is that for the blade coated sample the spherulites display the weakest PL emission but simultaneously the strongest light beam induced current. This finding is interpreted as evidence that photovoltaic action is more efficient at the spherulites, since radiative recombination and charge generation are mutually exclusive relaxation channels for the photogenerated electron-hole pairs. In Figure $3 b$ by comparison of integrated PL (top) and integrated current (bottom) it is clear that there is an anti-correlation between LBIC and PL. Indeed, the peak photocurrent is more than twice as large in the blade coated sample as compared to the spin coated one, this is especially relevant since spin coated devices $(350 \mathrm{~nm})$ are thinner than the doctor blade devices $(550 \mathrm{~nm})$. Alternatively, the PL is much less intense in the doctor blade sample than in the latter case despite the lower thickness. Strikingly, this implies that spherulitic formation, rather than being detrimental, can lead to better photovoltaic performance in hybrid perovskite films. Considering that the PL maximum is located at the domain boundaries as observed in Figure $3 \mathrm{a}$ (middle image) and that at this position the photocurrent does not decrease (bottom image) we can conclude that these defects do not compromise performance because they do not introduce additional non-radiative recombination pathways. As we will show, the macroscopic device performance also follows this trend.

\subsection{Crystallization mechanism}

Spherulites, aka spherical crystallites, are associated to a type of crystal growth common to many materials including minerals, synthetic polymers, organic compounds and inorganic salts. ${ }^{25}$ The growth starts at primary nuclei (Fig. 4a) and, unlike single crystal growth, the growth progresses in a space filling manner by branching from the radial crystallites branch in slightly different crystallographic orientations (Fig. 4b). The radially symmetric polycrystalline aggregates grow until the fibers impinge either an external boundary or another spherulite growing from a neighboring nucleus (Fig. 4c). The size of the spherulites depends on a complex interplay between the number of nuclei, the crystal growth velocity, solvent evaporation rate, diffusion coefficients of the different components, temperature, etc.
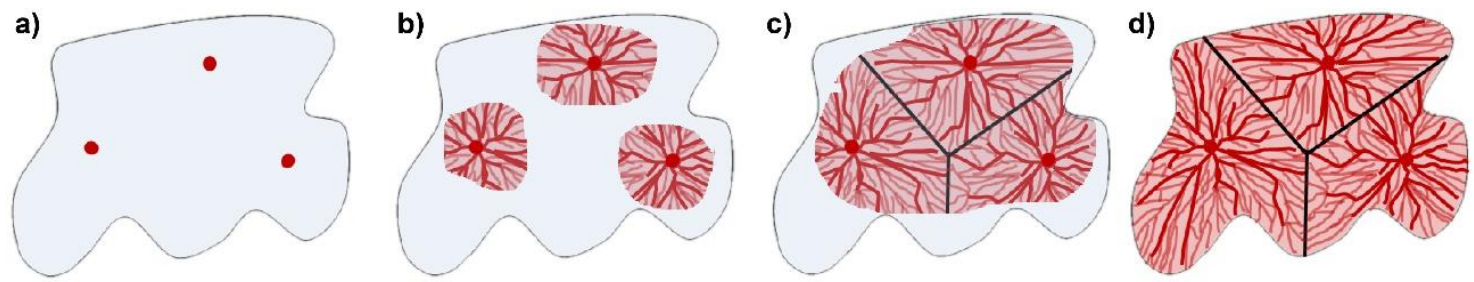

Figure 4: Different stages during spherulitic growth of the perovskite layer. 
The formation of ring-like or radial fibrillary structures is strongly related to the concentration of the solution. ${ }^{26}$ For unsaturated solutions, rings are formed due to the alternating occurrence of locally saturated solution and solute depleted solution as the spherical aggregate grows. Kajioka and co-workers have shown that the periodicity and amplitude of the ring structure is associated to the available volume, as well as substrate temperature. ${ }^{22}$ Saturated solutions, however, give rise to spherulitic textures more similar to those observed in polymers quenched from the melt or minerals crystallized from magmas, in which crystallites are radially oriented. The two key aspects for this to occur are a very high viscosity and a relatively slow crystal growth. In this work, we casted the precursor ink at $1.2 \mathrm{M}$, which corresponds to the saturation regime and, moreover, we kept the temperature $\sim 50^{\circ}$ below the boiling point of the solvents enabling a relatively slow solvent drying. The high viscosity granted by the saturation conditions clarifies why rings are not observed in the films formed by blade coating, as no solute depleted solvent is present. On the other hand, the need for relatively slow crystallization explains why we have not observed spherulite formation in the fast dried spin coated films, even if saturation concentrations are used. Looking at several blade coated samples, it is apparent that most of them have regions with spherulites and regions that appear homogeneous, or homogeneous regions between spherulites (like in Figure 3a).

The PL images serve to obtain further insights regarding crystal formation. We have not found differences in the PL intensity upon vertical or horizontal polarization of the laser beam (see supporting information), which suggest that either crystallites are not oriented radially or the degree of optical anisotropy is very small in the tetragonal phase, as it was also apparent in the dielectric tensor. ${ }^{27}$ Measurements of polarized optical microscopy (POM) did not reveal anisotropy either in transmission with cross-polarizer configuration, i.e. no Maltese cross was detectable.

As the $\mathrm{MAPbI}_{3}$ crystallites grow radially from a nucleus, they branch out filling the whole space. This has several consequences. First, no pin-holes appear in the perovskite layer. Second, slightly less crystalline regions may appear between radial crystallites. Some evidence of this is seen in the PL images as variations in PL intensity and PL maximum (Figure 3a). Impurities are expelled from the spherulite as it is created in order to form the crystallites (only a limited number of defects can be accommodated in the polycrystalline structure). These impurities accumulate at the spherulite boundaries. Evidence of the presence of self-passivated $\mathrm{PbI}_{2}$ between adjacent spherulites is seen in the SEM images (Figure 1) as brighter boundaries, and in the PL maps as strong PL shifts (Figure 3a). Finally, for many of the large spherulites for which enough resolution is available in the PL/LBIC experiment, an increase in the PL intensity and a decrease in photocurrent can be observed at the nucleus of the spherulite, which suggests that crystallite seeds might also be unconverted precursors or impurities.

In any case, judging by the local and macroscopic (discussed below) performances, it 
is clear that reaching a rapid saturation regime is beneficial to obtain a $\mathrm{MAPbI}_{3}$ morphology that will lead to high efficiency devices in one deposition step.

\subsection{Device preparation and characterization}

Photovoltaic devices were fabricated following the device architecture Glass/FTO/TiO 2 -cp/TiO 2 -ms/MAPbI $3 /$ Spiro-OMeTAD/Au and using optimum conditions to minimize byproducts according to the optical properties discussed above. Further details on the preparation can be found in the methods section., It is important to highlight, however, that the deposition of the $\mathrm{MAPbI}_{3}$ by doctor blade does not require any additional step such as the use of antisolvent to wash out undesired solvents present in the precursor solution or thermal or solvent annealing. Photovoltaic results are summarized in Table 1 and representative $J$ - $V$ curves are shown in Figure 5a. Further performance results using different solvent mixtures are shown as supporting information. Reference spin coated devices show efficiencies exceeding 16-17 \% in good agreement with reported devices fabricated in ambient conditions with a relative humidity of $45 \pm 10 \% .^{18}$

Table 1: Summary of photovoltaic performance parameters for champion devices fabricated under different conditions by doctor blade using GBL:DMSO (6:4) in comparison to reference cells fabricated by spin coating. Illuminated area: $0.11 \mathrm{~cm}^{2}$. In parenthesis, average values over $>10$ independent devices.

\begin{tabular}{|l|c|c|c|c|}
\hline & $\begin{array}{c}\boldsymbol{J}_{\boldsymbol{s c}} \\
\left(\mathbf{m A} / \mathbf{c m}^{\mathbf{2}}\right)\end{array}$ & $\begin{array}{c}\boldsymbol{V}_{\boldsymbol{o c}} \\
(\mathbf{V})\end{array}$ & $\begin{array}{c}\boldsymbol{F F} \\
(\boldsymbol{\%})\end{array}$ & $\begin{array}{c}\boldsymbol{P C E} \\
(\boldsymbol{\%})\end{array}$ \\
\hline Spin coating in Ambient & $20.9(20.0)$ & $1.06(1.03)$ & $73(72)$ & $16.2(16.0 \pm 0.4)$ \\
\hline Blade in Ambient & $20.6(19.3)$ & $1.01(0.97)$ & $75(73)$ & $15.6(13.7 \pm 0.6)$ \\
\hline Blade in Dry box & $22.9(22.3)$ & $1.04(1.00)$ & $77(72)$ & $18.0(16.1 \pm 1.2)$ \\
\hline
\end{tabular}

Conditions: R.H. $=45 \pm 10 \%$, Dry box: dry air with R.H. $<0.01 \%$.

Similarly, we also prepared devices by doctor blade using the optimum ink in ambient conditions at R.H. of $\sim 45 \%$. Maximum efficiencies of $15.6 \%$ are obtained with $V_{o c} \approx 40 \mathrm{mV}$ lower than samples obtained by spin coating with similar photocurrent densities and filling factors (FFs). The reduced efficiency compared to the spin coated devices is related to the chemical species observed in the absorption spectra (Figure 1b) that lead to recombination of carriers. In order to avoid the presence of undesirable water molecules during the perovskite deposition, the doctor blade setup was placed in a dry air environment (R.H. $<0.01 \%)$. The devices fabricated under these conditions show a considerably higher efficiency leading to a record efficiency of $18.0 \%$ for devices fabricated by doctor blade using non-toxic solvents. $J$ - $V$ curves of selected devices are shown in Figure 5a. As it can be observed, perovskites with spherulitic morphology show higher photocurrent and FF than the spin coated devices. Photocurrents are in good agreement with the integrated photocurrent extracted from EQE measurements, 
see supporting information. Figure $5 b$ shows statistical data of efficiencies of devices with their perovskite layer deposited under dry conditions. The average efficiency over 45 devices is $16.1 \%$, ranging efficiencies from $14 \%$ up to $18.0 \%$. It was observed that the dispersion of the data was related to the ambient humidity during deposition of the compact and mesoporous layers of $\mathrm{TiO}_{2}$. Unfortunately, the deposition techniques used to manufacture $\mathrm{TiO}_{2}$ cannot be transferred to the dry box due to our experimental limitations. In general, the best efficiencies were obtained when the $\mathrm{TiO}_{2}$ layers were deposited under low humidity conditions (R.H. <35\%). Therefore, we can conclude that doctor blade deposition technique for perovskite deposition is robust.
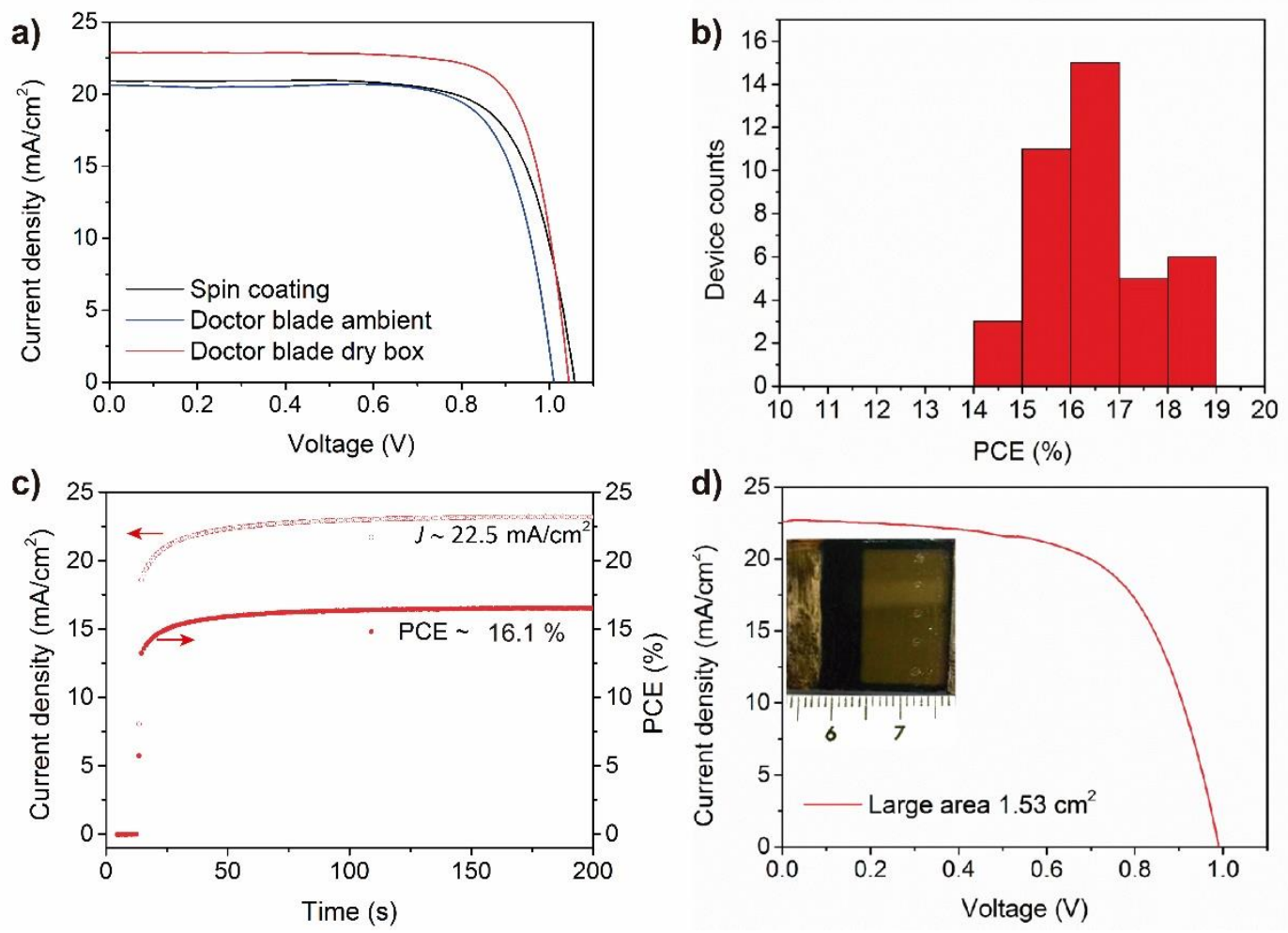

Figure 5: a) $J-V$ curves of devices fabricated under different conditions: spin coating and doctor blade (GBL:DMSO, 6:4), measurement carried out under reverse bias at 50 $\mathrm{mV} / \mathrm{s}$ at 1 sun illumination. b) Statistical efficiency data of 45 devices fabricated by doctor blade conditions under dry air. c) Stabilized efficiency measured at maximum power point tracking under 1 sun illumination conditions. d) Representative illumination $J-V$ curve of device fabricated with an active area of $1.53 \mathrm{~cm}^{2}$ measured with same conditions as in a).

Hysteresis in the $J-V$ curves was observed for all doctor bladed and spin coated devices, see Supporting Information. We point out that it has been previously reported that hysteretic behavior in the $J-V$ curve is related to the presence of ion migration towards the external interfaces in the perovskite. ${ }^{28,}{ }^{29}$ For example, Sargent et al. have recently canceled hysteresis by chemically modifying the $\mathrm{TiO}_{2}$ electron selective layer. ${ }^{2}$ 
However, modification of the external layers is outside the scope of this work and thus, further optimization of the external contacts may lead to higher efficiencies. In order to test the stability, devices were tracked at the maximum power point as a function of time (Figure 5c). The dynamic effects leading to hysteresis are also observed with photocurrent stabilizing after the initial $50 \mathrm{~s}$ to provide stabilized efficiencies of around $16.1 \%$. Similarly, degradation experiments following adequate protocols are beyond the scope and have not been carried out due to the lack of adequate equipment to promote degradation under well controlled conditions. ${ }^{30}$ However, we can state that in general devices were as stable as spin coated devices prepared and stored under similar ambient conditions, see supporting information.

Finally, to prove the scalability of doctor blade, large area devices were produced and measured. Table 2 shows a summary of average photovoltaic parameters fabricated with device areas ranging from 0.23 to $1.53 \mathrm{~cm}^{2}$. The efficiencies of larger cells are slightly lower than small record devices (14.82\%). Surprisingly, in all cases the photovoltaic parameters do not exhibit a clear dependence with the active area and it seems to depend more on other factors such as the reproducibility of the perovskite deposition or the crystallization processes. Reduced $F F$ are expected in comparison to small area devices due to the increased series resistance of large area devices where no special engineering layouts are employed. Figure $5 \mathrm{~d}$ shows a representative $J-V$ curve of a device with a 2.2 $\mathrm{cm}^{2}$ active area measured with a mask of $1.53 \mathrm{~cm}^{2}$, an image of the device is shown in the inset. In any case, the efficiencies for large area devices are within the highest reported for perovskite devices for one step and use of non-toxic solvents, in agreement with the small proportion of pinholes observed that slightly reduce the $V_{o c}$ and $F F{ }^{16}$

Table 2: Summary of average photovoltaic performance parameters for devices fabricated with different active areas.

\begin{tabular}{|l|c|c|l|l|l|}
\hline Area & $\begin{array}{c}\boldsymbol{I}_{\text {sc }} \\
(\mathbf{m A )}\end{array}$ & $\begin{array}{c}\boldsymbol{J}_{\boldsymbol{s c}} \\
\left(\mathbf{m A} / \mathbf{c m}^{2}\right)\end{array}$ & $\begin{array}{l}\boldsymbol{V}_{\boldsymbol{o c}} \\
(\mathbf{V})\end{array}$ & $\begin{array}{l}\boldsymbol{F F} \\
(\boldsymbol{\%})\end{array}$ & $\begin{array}{l}\boldsymbol{P C E} \\
(\boldsymbol{\%})\end{array}$ \\
\hline $0.23 \mathrm{~cm}^{2}$ & 5.22 & 22.7 & 0.96 & 67 & $14.8 \pm 1.3$ \\
\hline $0.56 \mathrm{~cm}^{2}$ & 12.9 & 22.5 & 0.99 & 62 & $14.0 \pm 1.6$ \\
\hline $1.53 \mathrm{~cm}^{2}$ & 34.7 & 22.7 & 0.99 & 63 & $14.2 \pm 1.5$ \\
\hline
\end{tabular}

\section{Conclusions}

In this work, we presented a simplified method to produce highly crystalline perovskite films using doctor blade in combination with non-toxic solvents. The process follows a spherulitic growth by working in the supersaturation regime to form highly crystalline perovskite layers. Spherulitic growth is shown to be beneficial for the device performance since chemical defects confined at the grain boundaries of the spherulites do not have a negative impact on charge extraction. Very importantly, the method does not require additional steps like antisolvent addition or solvent annealing to obtain adequate morphology, which clearly represents a great advantage towards 
commercialization of this technology.

\section{Methods}

\subsection{Materials and precursor solutions}

All materials were used as received: FTO glasses $(25 \times 25 \mathrm{~mm}$, Pilkington TEC15, $\sim 15 \Omega / \mathrm{sq}$ resistance), $\mathrm{TiO}_{2}$ paste (Dyesol 30NRD, $300 \mathrm{~nm}$ average particle size), $\mathrm{CH}_{3} \mathrm{NH}_{3} \mathrm{I}$ (DYESOL), $\mathrm{PbI}_{2} \quad$ (TCI, $99.99 \%$ ), titanium diisopropoxidebis (acetylacetonate) (75\% in isopropanol, Sigma-Aldrich), spiro-OMeTAD (Merck). The perovskite precursor solution was prepared by mixing GBL:DMSO solutions with MAI and $\mathrm{PbI}_{2}(1: 1 \mathrm{~mol} \%)$. GBL:DMSO ratio was modified as required for optimization. In the best conditions concentration of $1.2 \mathrm{M}$ and $6: 4$ volume ratio is used: $\mathrm{PbI}_{2} 553.2 \mathrm{mg}$, MAI $190.8 \mathrm{mg}$, GBL $600 \mu \mathrm{L}$, DMSO $400 \mu \mathrm{L}$. The spiro-OMeTAD solution was prepared by dissolving in $1 \mathrm{~mL}$ of chlorobenzene $72.3 \mathrm{mg}$ of $\left(2,2^{\prime}, 7,7^{\prime}\right.$-tetrakis( $\mathrm{N}, \mathrm{N}^{\prime}$-dip-methoxyphenylamine)-9,9'-spirobifluorene), $28.8 \mu \mathrm{L}$ of 4-tert-butylpyridine, and 17.5 $\mu \mathrm{L}$ of a stock solution of $520 \mathrm{mg} / \mathrm{mL}$ of lithium bis- (trifluoromethylsulfonyl) imide in acetonitrile.

\subsection{Fabrication of MAPbI 3 photovoltaic devices}

Prior to the deposition of $\mathrm{TiO}_{2}$ compact layer, the FTO substrate was partially etched with zinc powder and $\mathrm{HCl}(2 \mathrm{M})$ and cleaned by ultra-sonic bath in Hellmanex detergent, rinsed with Milli-Q water and in a solution of ethanol: isopropanol (1:1 v/v). The substrates were treated in a $\mathrm{UV}-\mathrm{O}_{3}$ cleaner for $10 \mathrm{~min}$ prior to deposition of the $\mathrm{TiO}_{2}$ compact layer. $\mathrm{TiO}_{2}$ compact layer was deposited by aerosol spray pyrolysis at 450 ${ }^{\circ} \mathrm{C}$, using a commercial titanium diisopropoxide bis(acetylacetonate) solution (75\% in 2propanol, Sigma-Aldrich) diluted in ethanol (1:9, v/v) as precursor, with oxygen as carrier gas and spraying a total volume of $5 \mathrm{~mL}$ (approx.), performed by 3 steps spraying of $6 \mathrm{~s}$ each one and waiting $30 \mathrm{~s}$ between steps. To form the mesoporous $\mathrm{TiO}_{2}$ layer, a paste of $\mathrm{TiO}_{2}$ (Dyesol 30NRD) is diluted in ethanol (1:5, weight ratio) and was spin-coated at $2000 \mathrm{rpm}$ for $10 \mathrm{~s}$. The sample is initially heated at $100^{\circ} \mathrm{C}$ for $10 \mathrm{~min}$ followed by a sintering step at $500^{\circ} \mathrm{C}$ for $30 \mathrm{~min}$ under room atmosphere. The perovskite precursor solution $(50 \mu \mathrm{L})$ was coated on a doctor blade coater (Zehntner automatic film applicator coater, model ZAA 2300) with substrate heated at $150{ }^{\circ} \mathrm{C}$, at a blade speed of $1 \mathrm{~mm} / \mathrm{s}$ and a gap between blade and substrate of $150 \mu \mathrm{m}$. The perovskite dark phase formed within the first few seconds. Perovskite precursor deposition was performed either in ambient conditions (R.H. $=45 \pm 10 \%$ ) or in a dry chamber containing dry air (R.H. $<0.01 \%$ ). The Spiro-OMeTAD precursor was prepared by mixing $72.3 \mathrm{mg}$ of (2,2',7,7'-tetrakis(N,N'-di-pmethoxyphenylamine)-9,9'-spirobifluorene), $28.8 \mu \mathrm{L}$ of 4 tert-butylpyridine and $17.5 \mu \mathrm{L}$ lithium bis-(trifluoromethylsulfonyl) imide in acetonitrile (from a stock solution of $520 \mathrm{mg} / \mathrm{mL}$ ) and $1 \mathrm{ml}$ of Chlorobenzene. The perovskite films were then covered with $50 \mu \mathrm{L}$ of the HTL solution by spin coating at $4000 \mathrm{rpm}, 800$ 
$\mathrm{rpm} / \mathrm{s}$ of acceleration for $30 \mathrm{~s}$. Finally, $60 \mathrm{~nm}$ of gold was thermally evaporated on top of the device as a back contact, using a commercial Univex 250 chamber, in the Oerlikon Leybold Vacuum chamber.

\subsection{Film and Device characterization}

The film morphology was analysed with a field-emission scanning electron microscope (JEOL 7001F), XRD. Photovoltaic devices were characterized using an Abett Solar simulator equipped with 1.5 AM filter. The light intensity was adjusted to100 $\mathrm{mWcm}^{-2}$ using a calibrated Si solar cell. Devices were measured using a mask with different sizes as described in the manuscript.

Photoluminescence (PL) and light-beam induced current (LBIC) experiments were measured co-locally (simultaneously) using a $633 \mathrm{~nm}$ He-Ne laser in a WITec alpha 300 RA+ confocal setup. We employed a $40 \mathrm{X}$ magnification objective with a glass correction collar to measure through the glass substrate of the perovskite devices (glass correction set to $2.0 \mathrm{~mm}$ ). The focused laser spot on the perovskite film was less than 10 microns in size. Due to the large PL efficiency of the MAPI we employed laser powers of the order of a few $\mu \mathrm{W}$ ( $<50 \mu \mathrm{W}$ in all cases) to collect the PL without saturation of the CCD camera and to avoid the photodegradation of the film. The acquisition time was set to $50 \mathrm{~ms}$ per point. The PL and photocurrent images typically consisted of $2 \times 2$ $\mathrm{mm}^{2}$ regions analyzed in lateral steps of $20 \mu \mathrm{m}$, thus collecting a total of 10,000 spectra per image. LBIC was measured simultaneously at the same time as the PL measurement was taken, using the PL excitation laser also as the LBIC excitation source. The photocurrent was collected in short circuit, current-voltage amplified close to the sample, and then processed using the WITec electronics through an auxiliary channel. The analysis was performed using WITec Project FOUR software.

\section{Supporting Information.}

Photovoltaic optimization parameters, , additional SEM images, polarized PL images of spherulites of different dimensions, and further $J$ - $V$ curves.

\section{Acknowledgements}

We greatly thank financial support by the Spanish Ministerio de Economía y Competitividad (MINECO) under projects MAT2016-76892-C3-1-R, MAT2015-70850P (HIBRI2), Ramón y Cajal Fellowship number RYC-2014-16809 and through Grant No. SEV-2015-0496 in the framework of the Spanish Severo Ochoa Centre of Excellence (ICMAB). X.X. and Z.B. would like to thank the Project on the Collaborative Innovation and Environmental Construction Platform of Guangdong Province (2014A050503051). We also thank the University Jaume I for financial support with the project (UJI-B2017-32) and its Serveis Centrals (SCIC) are acknowledged for the SEM measurement assistance. Finally, the authors at ICMAB would like to acknowledge financial support from the European Research Council 
(ERC) under grant agreement no. 648901.

\section{References}

1. M. Saliba, T. Matsui, K. Domanski, J.-Y. Seo, A. Ummadisingu, S. M. Zakeeruddin, J.-P. Correa-Baena, W. R. Tress, A. Abate, A. Hagfeldt and M. Grätzel, Science, 2016, 354, 206-209.

2. H. Tan, A. Jain, O. Voznyy, X. Lan, F. P. García de Arquer, J. Z. Fan, R. Quintero-Bermudez, M. Yuan, B. Zhang, Y. Zhao, F. Fan, P. Li, L. N. Quan, Y. Zhao, Z.-H. Lu, Z. Yang, S. Hoogland and E. H. Sargent, Science, 2017, 355, 722-726

3. NREL Efficiency Chart, https://www.nrel.gov/pv/assets/images/efficiencychart.png.

4. N. Ahn, D.-Y. Son, I.-H. Jang, S. M. Kang, M. Choi and N.-G. Park, J. Am. Chem. Soc., 2015, 137, 8696-8699.

5. N. J. Jeon, J. H. Noh, Y. C. Kim, W. S. Yang, S. Ryu and S. I. Seok, Nat. Mater., 2014, 13, 897-903.

6. Z. Xiao, Q. Dong, C. Bi, Y. Shao, Y. Yuan and J. Huang, Adv. Mater., 2014, 26, 6503-6509.

7. M. Yang, Z. Li, M. O. Reese, O. G. Reid, D. H. Kim, S. Siol, T. R. Klein, Y. Yan, J. J. Berry, M. F. A. M. van Hest and K. Zhu, Nat. Energy, 2017, 2, 17038.

8. Z. Yang, C.-C. Chueh, F. Zuo, J. H. Kim, P.-W. Liang and A. K. Y. Jen, $A d v$. Energy Mater., 2015, 5, 1500328-n/a.

9. Y. Deng, E. Peng, Y. Shao, Z. Xiao, Q. Dong and J. Huang, Energy Environ. Sci., 2015, 8, 1544-1550.

10. Y. Deng, Q. Wang, Y. Yuan and J. Huang, Materials Horizons, 2015, 2, 578583.

11. Y. Deng, Q. Dong, C. Bi, Y. Yuan and J. Huang, Adv. Energy Mater., 2016, 6, 1600372-n/a.

12. F. Mathies, T. Abzieher, A. Hochstuhl, K. Glaser, A. Colsmann, U. W. Paetzold, G. Hernandez-Sosa, U. Lemmer and A. Quintilla, Journal of Materials Chemistry A, 2016, 4, 19207-19213.

13. T. Qin, W. Huang, J.-E. Kim, D. Vak, C. Forsyth, C. R. McNeill and Y.-B. Cheng, Nano Energy, 2017, 31, 210-217.

14. Z. Bi, Z. Liang, X. Xu, Z. Chai, H. Jin, D. Xu, J. Li, M. Li and G. Xu, Sol. Energy Mater. Sol. Cells, 2017, 162, 13-20.

15. J. E. Bishop, D. K. Mohamad, M. Wong-Stringer, A. Smith and D. G. Lidzey, Scientific Reports, 2017, 7, 7962.

16. J. Wang, F. Di Giacomo, J. Brüls, H. Gorter, I. Katsouras, P. Groen, A. J. 
Janssen René, R. Andriessen and Y. Galagan, Solar RRL, 2017, 1, 1700091.

17. S. Rahimnejad, A. Kovalenko, S. Martí-Forés, C. Aranda and A. Guerrero, ChemPhysChem, 2016, 17, 2795.

18. C. Aranda, C. Cristobal, L. Shooshtari, C. Li, S. Huettner and A. Guerrero, Sustainable Energy \& Fuels, 2017, 1, 540 - 547.

19. J. Li, Q. Dong, N. Li and L. Wang, Adv. Energy Mater., 2017, DOI: 10.1002/aenm.201602922, 1602922-n/a.

20. W. S. Yang, J. H. Noh, N. J. Jeon, Y. C. Kim, S. Ryu, J. Seo and S. I. Seok, Science, 2015, 348, 1234-1237.

21. J. Xu, B.-H. Guo, G.-Q. Chen and Z.-M. Zhang, Journal of Polymer Science Part B: Polymer Physics, 2003, 41, 2128-2134.

22. H. Kajioka, M. Hikosaka, K. Taguchi and A. Toda, Polymer, 2008, 49, $1685-$ 1692.

23. Q. Chen, H. Zhou, T.-B. Song, S. Luo, Z. Hong, H.-S. Duan, L. Dou, Y. Liu and Y. Yang, Nano Lett., 2014, 14, 4158-4163.

24. L. Contreras-Bernal, C. Aranda, M. Valles-Pelarda, T. T. Ngo, S. Ramos-Terrón, J. J. Gallardo, J. Navas, A. Guerrero, I. Mora-Seró, J. Idígoras and J. A. Anta, J. Phys. Chem. C, 2018, 122, 5341-5348.

25. H. D. Keith and F. J. Padden, J. Appl. Phys., 1963, 34, 2409-2421.

26. A. G. Shtukenberg, Y. O. Punin, E. Gunn and B. Kahr, Chem. Rev. (Washington, DC, U. S. ), 2012, 112, 1805-1838.

27. A. M. A. Leguy, P. Azarhoosh, M. I. Alonso, M. Campoy-Quiles, O. J. Weber, J. Yao, D. Bryant, M. T. Weller, J. Nelson, A. Walsh, M. van Schilfgaarde and P. R. F. Barnes, Nanoscale, 2016, 8, 6317-6327.

28. J. Carrillo, A. Guerrero, S. Rahimnejad, O. Almora, I. Zarazua, E. Mas-Marza, J. Bisquert and G. Garcia-Belmonte, Adv. Energy Mater., 2016, 6, 1502246.

29. S. Ravishankar, O. Almora, C. Echeverría-Arrondo, E. Ghahremanirad, C. Aranda, A. Guerrero, F. Fabregat-Santiago, A. Zaban, G. Garcia-Belmonte and J. Bisquert, J. Phys. Chem. Lett., 2017, 8, 915-921.

30. M. Saliba, M. Stolterfoht, C. M. Wolff, D. Neher and A. Abate, Joule, 2018, 2, 1019-1024. 